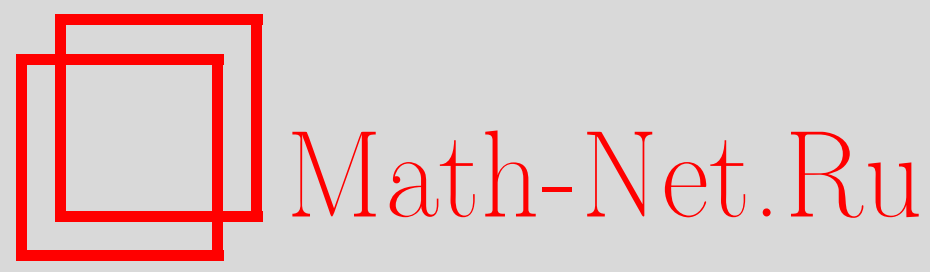

А. Г. Карапетян, О значениях случайных полиномов в окрестности единичной окружности, Матем. заметки, 1998, том 63, выпуск 1, 142-145

DOI: https://doi.org/10.4213/mzm1258

Использование Общероссийского математического портала Math-Net.Ru подразумевает, что вы прочитали и согласны с пользовательским соглашением http://www.mathnet.ru/rus/agreement

Параметры загрузки:

IP : 54.198 .64 .247

26 апреля 2023 г., 18:08:39 


\section{О ЗНАЧЕНИЯХ СЛУЧАЙНЫХ ПОЛИНОМОВ В ОКРЕСТНОСТИ ЕДИНИЧНОЙ ОКРУЖНОСТИ}

\section{А. Г. Карапетян}

Для независимых случайных величин $r_{0}, \ldots, r_{n-1}$, каждая из которых равна +1 или -1 с вероятностью $1 / 2$, обозначим через $P(u)$ вероятность:

$$
P(u)=P_{n}(u)=\mathrm{P}\left(\min _{x \in \mathbb{T}}\left|\sum_{j=0}^{n-1} r_{j} \exp (i j x)\right|>u\right), \quad u \geqslant 0 .
$$

Дж. Литтлвуд [1] предположил, что $P(\varepsilon \sqrt{n}) \rightarrow 0$ при $n \rightarrow \infty$ для любого $\varepsilon>0$. Б. С. Кашин [2] доказал эту гипотезу, причем рассмотрел минимум значений полинома не на всем торе $\mathbb{T}$, a на некотором подмножестве точек из отрезка $[0, \pi]$. Точнее, он установил, что при $n \rightarrow \infty$

$$
\mathrm{P}\left(\inf _{\nu=1,2, \ldots}\left|Q\left(\theta_{\nu}\right)\right|>n^{1 / 2}(\log n)^{-1 / 3}\right) \rightarrow 0
$$

где $\theta_{\nu}=2 \pi \cdot 2^{-\nu}$ при $\nu=1,2, \ldots$, а

$$
Q(x)=\sum_{j=0}^{n-1} r_{j} e^{i j x}
$$

А. М. Одлыжко показал, что $P\left(n^{1 / 3+\varepsilon}\right) \rightarrow 0$ при любом $\varepsilon>0$ и $n \rightarrow \infty$, и предположил, что при больших $n$ и всяком $\varepsilon>0$ для большинства полиномов

$$
G(x)=\sum_{j=0}^{n-1} \pm \exp (i j x)
$$

выполнена оценка

$$
\min _{x \in \mathbb{T}}|G(x)|<n^{-1 / 2+\varepsilon} .
$$

Эта гипотеза была доказана С. В. Конягиным [3], установившим, что $P\left(n^{-1 / 2+\varepsilon}\right) \rightarrow 0$ для любого $\varepsilon>0$ при $n \rightarrow \infty$. Далее, в [4] была доказана

Теорема. Пусть $\xi, \xi_{j}, j=0, \ldots, n-1,-$ действительные независимые одинаково распределенные случайные величины с конечными третьими абсолютными моментами и с нулевыми средними, удовлетворяющие условию $\mathrm{M} \xi^{2}>0$. Тогда для любого $\varepsilon \in(0,1)$ nрu $n>\left(16 C_{0}(\xi)\right)^{9936 / \varepsilon^{3}}$

$$
\mathrm{P}\left(\min _{x \in \mathbb{T}}\left|\sum_{j=0}^{n-1} \xi_{j} \exp (i j x)\right|>n^{-1 / 2+\varepsilon}\right) \leqslant \frac{1}{n^{\varepsilon^{2} / 180}},
$$

әде константа $C_{0}(\xi)$, зависящая только от $\xi$, определена в [4], а $\mathrm{M} \xi^{2}$ - второй момент случайной величины $\xi_{j}$.

(Случай $\mathrm{M} \xi^{2}=0$ является тривиальным, и при этом оценка вероятности в теореме, очевидно, справедлива для любого $n \in \mathbb{N}$.)

Работа выполнена при финансовой поддержке Российского фонда фундаментальных исследований, грант № 96-01-00378. 
Затем С. В. Конягиным и В. Шлагом в [5] было доказано, что для любого $\varepsilon>0$ и любой функции $\Phi \in H^{\sigma}[0,1] \backslash\{0\}, \sigma \in(1 / 2,1]$, для независимых случайных величин $r_{0}, \ldots, r_{n-1}$, имеющих радемахеровское или стандартное нормальное распределение, справедливо неравенство

$$
\varlimsup_{n \rightarrow \infty} \mathrm{P}\left(\left\{\min _{z \in \mathbb{C}:|| z|-1|<\varepsilon n^{-2}}\left|\sum_{j=0}^{n-1} r_{j} \Phi\left(\frac{j}{n}\right) z^{j}\right|<\varepsilon n^{-1 / 2}\right\}\right) \leqslant C \varepsilon,
$$

где $C$ - константа, зависящая от $\Phi$, а $H^{\sigma}$ - это гёльдеровский класс действительных функций порядка $\sigma$ на $[0,1]$.

Нашей целью было доказать это утверждение для более шшрокого класса случайных величин.

ОПРЕДЕЛЕНИЕ. Действительная случайная величина $\xi$ называется субгауссовской [6, с. 108], если она удовлетворяет условию

$$
\mathrm{M}\left(e^{\lambda \xi}\right) \leqslant e^{\tau^{2} \lambda^{2} / 2}, \quad-\infty<\lambda<\infty
$$

при некотором $\tau>0$.

Любая ограниченная случайная величина с нулевьм средним является субгауссовской величиной $[7$, с. 39]. В то же время, любая субгауссовская величина имеет нулевое среднее и конечный момент любого порядка [6, с. 108].

Доказана следующая теорема.

Теорема 1. Пусть $\xi, \xi_{j}, j=0, \ldots, n-1,-$ действительные независимые одинаково распределенные субаауссовские случайные величинь, удовлетворяющие условию $\mathrm{M}^{2}=$ $\mathrm{M} \xi_{j}^{2}>0$, и пусть $\Phi \in H^{\sigma}[0,1] \backslash\{0\}$, где $\sigma \in(1 / 2,1]$. Тогда для любого $\varepsilon>0$

$$
\varlimsup_{n \rightarrow \infty} \mathrm{P}\left(\left\{\min _{z \in \mathbb{C}:|| z|-1|<\varepsilon n^{-2}}\left|\sum_{j=0}^{n-1} \xi_{j} \Phi\left(\frac{j}{n}\right) z^{j}\right|<\varepsilon n^{-1 / 2}\right\}\right) \leqslant C \varepsilon,
$$

әде C - константа, зависящая от $\Phi$ и

СледСтвиЕ. Выполнено

$$
\varlimsup_{n \rightarrow \infty} \mathrm{P}\left(\left\{\min _{x \in \mathbb{T}}\left|\sum_{j=0}^{n-1} \xi_{j} \exp (i j x)\right|<\varepsilon n^{-1 / 2}\right\}\right) \leqslant C \varepsilon .
$$

Тем самым, на основании следствия из теоремы 1 мы получили, что с большой вероятностью

$$
\min _{x \in \mathbb{T}}\left|\sum_{j=0}^{n-1} \xi_{j} \exp (i j x)\right|
$$

по порядку не меньше $n^{-1 / 2}$.

Для доказательства теоремы 1 мы пользуемся теоремой Берри-Эссена [8, следствие 17.2], а также теоремой 8.4 из [8]. Решающую роль играют оценки характеристических функций $f_{x}, g_{x}$ соответственно векторов

$$
\frac{1}{\sqrt{n}}(\operatorname{Re} J(x), \operatorname{Im} J(x)), \quad \frac{1}{\sqrt{n}}\left(\operatorname{Re} J(x), \operatorname{Im} J(x), \operatorname{Re}\left(\frac{J^{\prime}(x)}{i n}\right), \operatorname{Im}\left(\frac{J^{\prime}(x)}{i n}\right)\right),
$$

где

$$
J(x)=\sum_{j=0}^{n-1} \xi_{j} \Phi\left(\frac{j}{n}\right) e^{i j x}
$$


(считаем $n$ достаточно большим):

$$
\sup _{\omega \in I_{1}}\left|f_{x}(\omega)\right|<\exp \left(-n^{\tau}\right), \quad I_{1}=\left\{\omega \in \mathbb{R}^{2}: n^{1 / 6-\tau / 2}<|\omega|<n^{1+\tau}\right\},
$$

$\sup _{(u, v) \in I_{2}}\left|g_{x}(u, v)\right|<\exp \left(-n^{\tau}\right), \quad I_{2}=\left\{(u, v), u, v \in \mathbb{R}^{2}: n^{1 / 6-\tau / 2}<|u|<n^{1+\tau},|v| \leqslant n^{\tau}\right\}$ для $\tau=(\sigma-1 / 2) / 10$ и точек $x$, удовлетворяюших условию

$$
\left|x-\frac{2 \pi h}{k}\right|>2 \pi n^{-1+\sigma / 20}
$$

для любых $h$ и $k, 0 \leqslant h \leqslant k-1,1 \leqslant k \leqslant H, H-$ константа, зависящая от $\Phi$.

Можно ожидать, что утверждение теоремы 1 будет справедливо при ослаблении условий на $\xi$; возможно, достаточно предположить, что $\mathrm{M}|\xi|^{3}<\infty$ и $\mathrm{M}^{2}>0$.

В работе [4] (основные результаты опубликованы также в [9]) для доказательства теоремы была предварительно получена асимптотическая оценка вероятности события

$$
\left(\left\{\operatorname{Re} T(x), \ldots, \operatorname{Re}\left(\frac{T^{(r-1)}(x)}{(i n)^{r-1}}\right), \operatorname{Im} T(x), \ldots, \operatorname{Im}\left(\frac{T^{(r-1)}(x)}{(i n)^{r-1}}\right)\right\} \in \Pi\right),
$$

где

$$
T(x)=\sum_{j=0}^{n-1} \xi_{j} \exp (i j x),
$$

$\xi_{j}$ - случайные величины такие же, как в условии теоремы [4], $r \in \mathbb{N}, 10 / \varepsilon<r<12 / \varepsilon$ для заданного $\varepsilon \in(0,1), x=x_{k}=2 \pi k / k_{0}, 0<k<k_{0}$, где $k_{0}$ - наибольшее простое, не превосходящее $n^{1-\varepsilon / 20}$, а П-произвольный $2 r$-мерный куб со стороной $h=n^{-1 / 2+\varepsilon / 2}$, причем $\complement[-H, H]^{2 r}$, $H=n^{1 / 2-\varepsilon /(5 r)}$.

В связи с этой оценкой возникла задача: получить асимптотическую оценку для вероятности $\mathrm{P}(T(x) \in \Pi)$ для двумерного квадрата П с максимальньм ослаблением условия на длину стороны $\Pi$, а также условия принадлежности П большому квадрату, и впоследствии распространить оценку со случая квадрата П на случай любого выпуклого множества $S$ с некоторыми ограничениями на расположение, на объем $S$ и на $x$. В этом направлении нам удалось получить следующее утверждение.

Теорема 2. Пусть $\xi, \xi_{j}, j=0, \ldots, n-1,-$ действительные независимые одинаково распределенные случайные величины с конечными третьими абсолютными моментами и с нулевыми средними, удовлетворяющие условию $\mathrm{M} \xi^{2}=\mathrm{M} \xi_{j}^{2}>0, u$

$$
T(x)=\sum_{j=0}^{n-1} \xi_{j} \exp (i j x) .
$$

Тогда для любого $\varepsilon \in(0,1 / 2]$, для $x=2 \pi p / q,(p, q)=1, n^{\varepsilon} \leqslant q \leqslant n^{1-\varepsilon}$, и любого випуклого мнохества $S$, удовлетворяюшего условиям $\operatorname{Vol} S \geqslant n^{-\rho(n)}, S \subset\left\{\omega:\|\omega\| \leqslant n^{1 / 2} H(n)\right\}$, где $\rho(n) \rightarrow \infty, \rho(n)=o(\ln n), H(n)=o(\sqrt{\ln n}), H(n) \rightarrow \infty$ при $n \rightarrow \infty$, имеем

$$
\mathrm{P}\{T(x) \in S\}=\frac{1}{\pi n \mathrm{M} \xi^{2}} \int_{S} \exp \left(-\frac{\|\tau\|^{2}}{n \mathrm{M} \xi^{2}}\right) d \tau \cdot(1+o(1)), \quad n \rightarrow \infty,
$$

причем остаточный член о(1) равномерен по $p, q, S$.

Было бы интересно исследовать сходимость случайных векторов

$$
\frac{1}{\sqrt{n}}\left(\sum_{j=0}^{n-1} \xi_{j} \cos (j x), \sum_{j=0}^{n-1} \xi_{j} \sin (j x)\right)
$$

к нормальному распределению при $n \rightarrow \infty$ в зависимости от скорости приближения числа $x /(2 \pi)$ рациональными.

Автор благодарит научного руководителя С. В. Конягина за полезные обсуждения и важные указания. 


\section{СПИСОК ЦИТИРОВАННОЙ ЛИТЕРАТУРЫ}

1. Littlewood J. E. // J. London Math. Soc. 1966. V. 41. P. 367-376. 2. Кашин Б. С. // Вестн. МГУ. Сер. 1. Матем., мех. 1987. № 5. С. 40-46. 3. Конягин С. В. // Матем. заметки. 1994. Т. 56. № 3. С. 80-101. 4. Карапетян А. Г. О минимуме модуля тригонометрических полиномов со случайными коэффициентами // Деп. ВИНИТИ. № 3330-B96. 5. Konyagin S. V., Schlag W. Lower bounds for the absolute value of random polynomials on a neighborhood // Trans. Amer. Math. Soc. (to appear). 6. Кахан Ж. Случайные функциональные ряды. М.: Наука, 1973. 7. Кашин Б. С., Сааклн А.А. Ортогональные ряды. М.: Наука, 1984. 8. Бхаттачария Р. Н., Ранга Р ао Р. Аппроксимация нормальным распределением и асимптотические разложения. М.: Наука, 1982. 9. Карапетян А. Г. // Матем. заметки. 1997. Т. 61. №3. С. 451-455.

Московский государственный университет им. М.В. Ломоносова 